\title{
La reestructuración de la red de oficinas en la banca española durante la crisis*
}

\author{
Lucio Fuentelsaz \\ Minerva González \\ Sergio Palomas \\ Universidad de Zaragoza
}

\section{Resumen}

Este trabajo trata de identificar los principales factores que explican la fuerte caída en la red de oficinas bancarias en España durante los últimos años. Nuestra aproximación es eminentemente descriptiva y a través de un análisis de descomposición de la varianza se evalúa la importancia relativa de cada uno de estos factores. Los resultados ponen de manifiesto que la caída en el volumen de negocio es el principal detonante de la reducción en la red. A continuación, y con una importancia relativa similar, debemos mencionar la corrección de las estrategias de crecimiento llevadas a cabo durante los años de bonanza, las diferencias en la misión y objetivos de las entidades y el reajuste de oficinas que se deriva de los procesos de fusión y adquisición. Sin embargo, factores como el desarrollo tecnológico o las diferencias en los niveles de eficiencia parecen haber tenido un peso marginal en dicha explicación.

Palabras clave: reestructuración de la red de oficinas, banca española, análisis de descomposición de la varianza, crisis.

Clasificación JEL: G21, L11, M21.

\begin{abstract}
This paper identifies the main factors that explain the strong reduction in the Spanish branching network in recent years. Our approach, essentially descriptive, uses a Variance Decomposition Analysis to assess the relative importance of different factors. The results show that the fall in the banking activity is the main trigger of the decrease in the banking network. Therewith, and with a similar relaftive importance, the correction of the growth strategies followed during flourishing years, the differences in the mission and objectives and the branching reduction following mergers and acquisitions should also be mentioned. On the contrary, factors such as technological development or the differences in efficiency levels seem to have had a secondary effect on the observed pattern.
\end{abstract}

Keywords: branching network restructuring, Spanish banking, variance decomposition analysis, crisis.

JEL classification: G21, L11, M21.

* La realización de este trabajo ha contado con la ayuda financiera del Ministerio de Economía y Competitividad y el FEDER (proyecto ECO2014-53904-R), así como del Gobierno de Aragón y el Fondo Social Europeo (grupos de investigación, S09). Los autores agradecen los comentarios y sugerencias de Jaime Gómez. 


\section{Introducción}

El sector bancario ha sufrido una transformación sin precedentes en los últimos años, tanto en España como en el resto de países de su entorno económico. En particular, el proceso desregulador que tuvo lugar durante los últimos lustros del pasado siglo supuso un cambio sustancial en las reglas de juego, que indujo a muchas empresas a crecer de forma acelerada, tanto dentro de sus mercados de actuación tradicionales como, con frecuencia, en nuevos mercados en los que no habían tenido presencia hasta ese momento. Téngase en cuenta que, al comienzo de aquel proceso, tanto las cajas de ahorro como las cooperativas de crédito veían limitada su actividad a un ámbito geográfico reducido, por lo que la eliminación de restricciones supuso un estímulo para muchas entidades, que intentan ampliar su ámbito de actuación, con el fin de prestar sus servicios en una buena parte del territorio español. La principal consecuencia de estos cambios fue una proliferación en el número de oficinas, que aumentó de forma ininterrumpida hasta el año 2008, momento en que los diferentes intermediarios ofrecían sus servicios a través de una red que superaba los 45.000 puntos de venta.

Sin embargo, la reciente crisis económica ha afectado de forma especialmente intensa al sector bancario, de modo que la mayoría de las entidades se han visto forzadas a replantear sus estrategias, lo que ha derivado en numerosas fusiones o en el cierre de buena parte de las oficinas existentes al comienzo de la crisis.

Aunque la expansión que tuvo lugar hasta finales de la pasada década ha sido suficientemente documentada por la literatura, tanto desde un punto de vista agregado (Delgado, Saurina y Townsend, 2008) como de forma individualizada, analizando la estrategia adoptada por determinadas entidades (Fuentelsaz, Gómez y Polo, 2004; Fuentelsaz, Gómez y Palomas, 2007), el proceso de reducción posterior en el número de oficinas, seguramente por su proximidad en el tiempo, ha sido mucho menos estudiado. Es cierto que en algunos trabajos se ha hecho referencia a la contracción en el tamaño de la red una vez desencadenada la crisis económica que se inicia en el año 2008 y a las consecuencias de dicha crisis en el sector, pero hasta el momento son más limitadas las investigaciones que han estudiado con detalle este proceso. En este sentido, deben destacarse los trabajos de Maudós $(2017,2018)$ que, en su intento por profundizar en las consecuencias del cierre de oficinas desde el punto de vista de la prestación de servicios financieros a las comunidades más desfavorecidas, analiza con detalle el volumen de población que ha perdido el acceso a los servicios bancarios, aproximando la exclusión financiera a través del número de municipios en los que no existe ninguna oficina bancaria.

En este trabajo tratamos de adoptar una perspectiva más amplia con el fin de ahondar en los factores que han tenido una mayor incidencia en el cierre de oficinas producido en estos últimos años. No cabe duda de que la respuesta a esta pregunta no resulta sencilla, ya que pueden ser numerosos los motivos que justifican dichos cierres. Es evidente que la caída en la demanda de los servicios ofrecidos por las entidades financieras o el importante cambio tecnológico que ha tenido lugar en la 
industria y que aconseja entidades de mayor dimensión con el fin de poder afrontar las cuantiosas inversiones necesarias para desarrollar dichas tecnologías constituyen argumentos importantes. Pero el comportamiento adoptado por las propias entidades, tanto antes de surgir las primeras dificultades como la respuesta ofrecida por las mismas en esos momentos iniciales, también forma parte de la explicación. Seguramente algunas habían crecido de forma excesiva y sus oficinas no habían sido capaces de lograr un tamaño suficiente, o lo habían hecho a costa de elevar en exceso sus costes operativos. Otras se encontraban inmersas en complejos proceso de fusión, o los inician al percibir las primeras dificultades, con la esperanza de que estas fusiones pudieran formar parte de la solución al problema. Nuestro análisis no apuesta, a priori, por ninguno de estos motivos como el principal factor determinante de la reestructuración, sino que trata de identificar, a través de un enfoque eminentemente descriptivo, cuáles de estos factores han podido resultar especialmente relevantes.

Con el fin de profundizar en este análisis, el resto del trabajo se estructura como sigue. La sección siguiente proporciona una primera aproximación a la evolución de la red de oficinas en España en el último cuarto de siglo, diferenciando según el tipo de entidad e identificando tres periodos temporales con comportamientos sustancialmente diferentes (1991-2000, 2000-2008 y 2008-1015). A continuación, y dado que en este trabajo se analiza el comportamiento de todas las entidades que operan en el segmento de banca minorista en España (considerando como tales aquellas que tienen al menos 10 oficinas en alguno de los años que forman parte de nuestro periodo muestral) analizamos la evolución de la red de oficinas de las principales entidades que operan en nuestro país. La sección cuarta identifica seis posibles factores que han podido incidir especialmente en la reestructuración de la red (caída de la demanda, evolución tecnológica, estrategias de crecimiento seguidas durante el periodo de bonanza, participación en fusiones y adquisiciones, diferencias en la misión o el modelo de negocio o grado de eficiencia), planteando a continuación un análisis de descomposición de la varianza con el fin de evaluar la importancia relativa de cada uno de estos factores. La última sección del trabajo sintetiza las principales conclusiones que se derivan del mismo.

\section{Evolución de la red de oficinas 1991-2015: una visión general}

Antes de analizar el comportamiento individual de las entidades bancarias que operan en España o la magnitud de la reestructuración de su red de oficinas, puede resultar de utilidad conocer brevemente su evolución en términos agregados. Con el fin de disponer de una perspectiva temporal suficientemente amplia, la evaluación que se lleva a cabo en esta sección tiene en cuenta el desarrollo del sector en el último cuarto de siglo, y más concretamente en el periodo 1991-2015. No obstante, es importante mencionar previamente que durante los años 70 y 80 del siglo pasado se produjeron una serie de cambios regulatorios importantes, orientados a liberalizar el sector e incrementar la rivalidad dentro del mismo y que probablemente desencadenan 
muchos de los movimientos posteriores. Entre estos cambios, cabe mencionar aspectos como la libertad de apertura de oficinas, la eliminación de restricciones a los tipos de interés de préstamos o depósitos, la reducción de las barreras a la entrada de bancos extranjeros o la supresión de coeficientes de inversión obligatorios ${ }^{1}$. Como consecuencia de todos estos cambios, a comienzos de los años noventa el sector puede considerarse razonablemente competitivo y las entidades comienzan a redefinir sus estrategias, una de cuyas manifestaciones más evidente es la búsqueda de nuevos mercados por parte de muchas de ellas, con el consiguiente efecto en el tamaño de la red de oficinas.

El Gráfico 1, que muestra el comportamiento de la red desde 1991, proporciona una primera aproximación a dicha evolución. En dicho gráfico se observa un crecimiento continuado hasta 2008, año en el que se alcanza un máximo histórico de 45.652 oficinas, lo que supone un crecimiento del 34 por 100 en relación con las 34.031 existentes en 1991. A partir de ese momento, el número de puntos de venta se reduce progresivamente hasta las 31.252 oficinas de 2015, lo que implica el cierre de aproximadamente una de cada tres oficinas en tan solo siete años. Como consecuencia de estos cierres, el tamaño de la red es en la actualidad inferior a la existente en 1991 (un 8,2 por 100 menor), a pesar de lo cual parece que el proceso de restructuración no ha finalizado ${ }^{2}$.

En la medida en que en la industria han participado tradicionalmente diferentes tipos de agente, cada uno de ellos con unas características y comportamiento diferentes, el Gráfico 2, así como el Cuadro 1, muestran su evolución por separado y ponen de manifiesto la existencia de patrones de comportamiento claramente diferenciados. En todo caso, y antes de continuar con el análisis, parece procedente realizar algunas puntualizaciones en relación con la información contenida en ambos. Así, es importante destacar en primer lugar que desde 2010 la mayor parte de las cajas de ahorros han comenzado un proceso de bancarización importante que ha llevado a la desaparición como tales de casi todas ellas ${ }^{3}$. En consecuencia, es discutible que en la actualidad debamos llamar a estas entidades cajas de ahorro. Dado que nuestro objetivo en este punto es incidir en la dispar evolución experimentada por los diferentes tipos de intermediarios y con el fin de evitar, en lo posible, problemas de terminología, diferenciamos las entidades en función de su asociación a las diferentes patronales que continúan existiendo en la actualidad. Hablamos de bancos o miembros AEB para las entidades integradas en la Asociación Española de Banca, miembros CECA (y, ocasionalmente, aun siendo conscientes del abuso de lenguaje que ello supone, cajas de ahorro) para las cajas tradicionales, y miembros UNACC para las cajas

\footnotetext{
${ }^{1}$ Un análisis detallado de las reformas que se han llevado a cabo en el sector bancario español puede verse en MALO DE MOLINA y MARTÍN-ACEÑA (2011).

2 Según el dato más reciente del Banco de España, en diciembre de 2016 había 28.807 oficinas operativas en territorio nacional. Esto supone un descenso del 7,8 por 100 tras el fin del horizonte temporal del estudio.

${ }^{3}$ Las dos únicas excepciones vienen dadas por dos pequeñas entidades, Caixa Ontinyent y Caixa de Ahorros de Pollensa, que han mantenido su estatus de cajas.
} 


\section{GRÁFICO 1}

EVOLUCIÓN DE LA RED DE OFICINAS BANCARIAS EN ESPAÑA

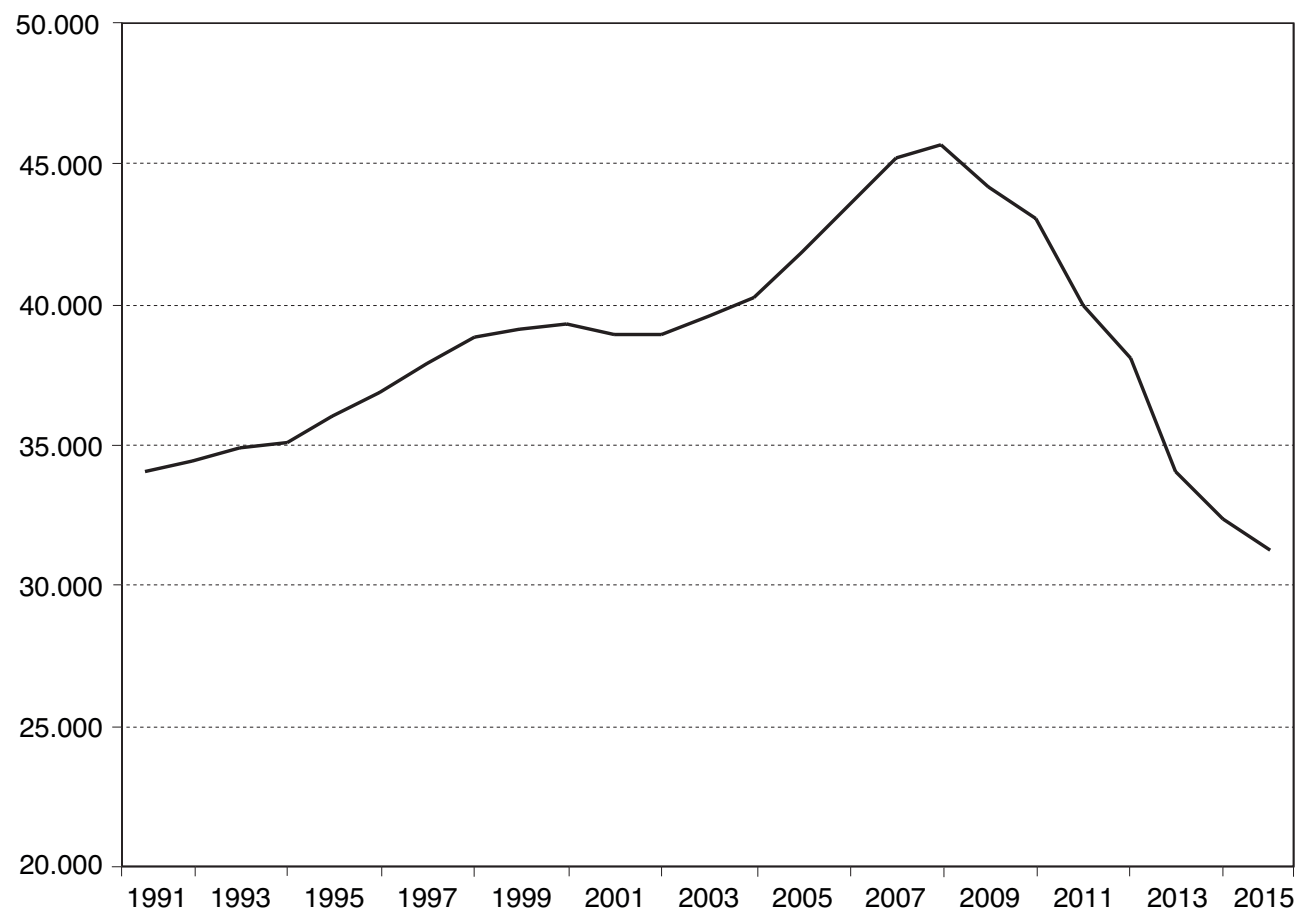

FUENTE: Elaboración propia.

rurales y las cooperativas de crédito ${ }^{4}$. En segundo lugar, durante la última década se han producido numerosas adquisiciones de entidades entre cajas de ahorro y bancos comerciales. Esto podría generar variaciones repentinas en el número de entidades de cada grupo que no se deben realmente a aperturas y cierres de oficinas, sino a traspasos de oficinas de un tipo de entidad a otro. Para evitar el impacto de estas variaciones en el análisis de la red, el número de oficinas de cada grupo se calcula de forma retrospectiva. Por ejemplo, cuando una caja adquiere un banco, se suman las oficinas de este banco a todas las observaciones de la caja en años anteriores, con independencia del momento del tiempo en el que tuvo lugar la adquisición.

Por último, indicar que dentro del periodo de análisis consideramos, a su vez, tres subperiodos, con el fin de identificar las principales tendencias que se producen en cada uno de ellos. El primer tramo, 1991-2000, representa la consolidación del

\footnotetext{
${ }^{4}$ Cajamar, el principal grupo cooperativo del país, ha dejado de ser miembro de UNACC, constituyendo la Asociación Empresarial de Entidades Cooperativas de Crédito (ASEMECC). Dado que durante la mayor parte de los años del horizonte temporal considerado ha estado integrada en UNACC y que en la actualidad mantiene su espíritu cooperativo, nos referiremos a esta entidad como miembro de este grupo a pesar de que en la actualidad ya no forma parte del mismo.
} 
sistema bancario español tras la compleción del proceso desregulador y antes de la introducción del euro como moneda única dentro de la Unión Económica y Monetaria ${ }^{5}$. Los quince años restantes se dividen en dos subperiodos de ocho y siete años respectivamente y cuyo punto de separación se sitúa en el inicio de la gran crisis económica que tiene lugar a partir de 2008. De esta forma, comparamos la evolución de la red de oficinas en el periodo de crecimiento económico comprendido entre 2000 y 2008, y la que tiene lugar una vez desencadenada la crisis. En definitiva, estos tres periodos recogen tres contextos económicos claramente diferentes en los que se ha desarrollado la actividad bancaria.

Una vez realizadas estas puntualizaciones y volviendo a la información contenida en el Gráfico 2 y el Cuadro 1, podemos constatar cómo en el caso de las entidades AEB la red de oficinas se reduce en una cuarta parte entre 1991 y 2015, con un comportamiento claramente diferenciado en los tres periodos considerados. Hasta el año 2000 la reducción tiene lugar de forma suave, a un ritmo del 0,5 por 100 anual, caída que se detiene entre 2000 y 2008 , para acelerarse a partir de este momento, con el cierre de casi el 25 por 100 de las oficinas en tan solo ocho años.

\section{GRÁFICO 2}

\section{EVOLUCIÓN DE OFICINAS POR TIPO DE ENTIDAD}

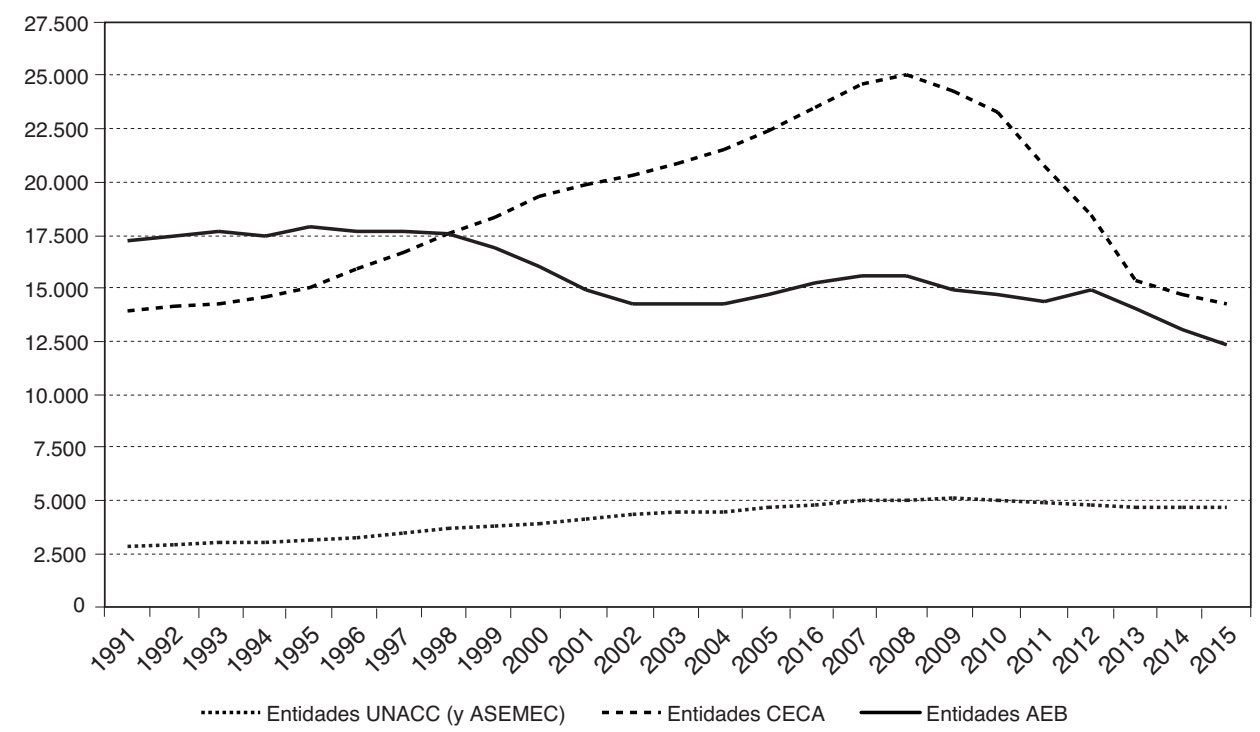

FUENTE: Elaboración propia a partir de datos AEB, CECA y UNACC.

${ }^{5}$ El euro nace el 1 de enero de 1999, momento en el que dicha moneda sustituye a la de los once países que se incorporan al mismo en la primera fase (Alemania, Austria, Bélgica, España, Francia, Irlanda, Italia, Luxemburgo, Países Bajos, Portugal y Finlandia). No obstante, la introducción física de las monedas y billetes no tiene lugar hasta el 1 de enero de 2002. 
En el caso de las entidades asociadas a la CECA el patrón es muy diferente. Desde la desregulación y hasta el comienzo de la crisis se observan tasas anuales de crecimiento elevadas, por encima del 3 por 100. Así, la red aumenta desde las 14.239 oficinas de 1991 hasta las 24.260 que operan al comienzo de la crisis, lo que supone un crecimiento superior al 56 por 100 en poco más de 15 años. Sin embargo, con el comienzo de crisis se observa un fuerte cambio de tendencia, con una tasa de cierres promedio del 7,3 por 100 anual, lo que supone volver a una cifra similar a la de 1991, esto es, 14.270 oficinas.

Finalmente, las entidades vinculadas a UNACC muestran un patrón similar al de las asociadas a la CECA hasta el comienzo de la crisis. Tras la desregulación crecieron a un ritmo acumulativo del 4 por 100 anual hasta el cambio de siglo $(3,3$ por 100 las entidades CECA), mientras que entre 2000 y 2008 lo hacen a una tasa del 3,1 por 100 anual, prácticamente la misma que en las entidades CECA. Es tras el estallido de la crisis cuando se detectan notables diferencias ya que mientras las antiguas cajas de ahorro cierran más del 40 por 100 de su red de oficinas, los cierres en las cooperativas son mucho más moderados: apenas el 1,2 por 100 cada año. Como consecuencia de ello, entre 1991 y 2015 la red de las entidades integradas dentro de la UNACC ha aumentado un 67 por 100, al pasar de 2.784 a 4.651 oficinas.

El Cuadro 1 completa la información proporcionada por el Gráfico 2, al recoger, tanto para todo el horizonte temporal considerado como para cada uno de los tres subperiodos, la variación total acumulada en la red de oficinas (Panel A) y las tasas de variación anual (Panel B). Esta evolución se analiza tanto de forma global como distinguiendo según el tipo de intermediario, esto es, banca comercial (miembros AEB), cajas de ahorro (miembros CECA) y cajas rurales (miembros UNACC).

\section{CUADRO 1 \\ VARIACIÓN EN EL NÚMERO DE OFICINAS BANCARIAS}

(En \%)

\begin{tabular}{|c|c|c|c|c|c|c|c|c|}
\hline & \multicolumn{3}{|c|}{ PANEL A: Variación acumulada } & \multicolumn{4}{c|}{ PANEL B: Variación anual } \\
\cline { 2 - 9 } & Total & AEB & CECA & UNACC* & Total & AEB & CECA & UNACC* \\
\hline $1991-2015$ & $-8,2$ & $-27,5$ & 0,22 & 67,1 & $-0,4$ & $-1,3$ & 0,01 & 2,2 \\
\hline $1991-2000$ & 15,4 & $-4,0$ & 33,5 & 41,7 & 1,6 & $-0,5$ & 3,3 & 4,0 \\
\hline $2000-2008$ & 16,3 & 0,2 & 27,7 & 27,8 & 1,9 & 0,02 & 3,1 & 3,1 \\
\hline $2008-2015$ & $-31,5$ & $-24,6$ & $-41,2$ & $-7,8$ & $-5,3$ & $-4,0$ & $-7,3$ & $-1,2$ \\
\hline
\end{tabular}

NOTA: Incluye también las entidades pertenecientes a ASEMECC

FUENTE: Anuarios estadísticos de la AEB, CECA y UNACC. Memoria de Cajamar. 


\section{Análisis individual}

La sección previa ha puesto de manifiesto la existencia de importantes diferencias tanto en las tasas de crecimiento de la red de oficinas según el periodo considerado como en lo que respecta a la heterogeneidad en el comportamiento de los distintos grupos de intermediarios. Con el fin de evaluar si dentro de un mismo grupo la situación es más homogénea, a continuación se consideran las principales entidades que operan actualmente en nuestro país. Para no distorsionar en exceso nuestro análisis, únicamente tendremos en cuenta el periodo 2000-2015, ya que al agregar retrospectivamente las oficinas cuando se producen fusiones o adquisiciones, las cifras se ven fuertemente afectadas por este tipo de operaciones. Del mismo modo y con el fin de no extender en exceso el análisis, únicamente se consideran las principales entidades del país, entendiendo como tales aquellas que durante los años considerandos han tenido, en promedio, un número de oficinas superior a $200^{6}$.

En primer lugar se muestra la evolución de las principales entidades integradas actualmente en la Asociación Española de Banca ${ }^{7}$. Por lo que respecta a los dos principales bancos del país (Gráfico 3), ambos siguen un patrón relativamente similar, con una reducción de sus oficinas superior a la de sus competidores, especialmente durante el primer tramo considerado. Téngase en cuenta, no obstante, que la fuerte caída que tiene lugar en ambos casos en 2001 y 2002 (y que conlleva que en el primer subperiodo ambos grupos reduzcan, en contra de la tendencia general, su red), viene dada por la reestructuración que se deriva como consecuencia de las fusiones que dan lugar a BBVA y Santander Central Hispano producidas a finales del pasado siglo. A partir de ese momento ambas entidades incrementan su tamaño hasta 2008, observándose una fuerte caída desde entonces, superior al 25 por 100 en ambos casos.

Según muestra el Gráfico 4, también es similar el comportamiento de Sabadell ${ }^{8}$ y del Grupo Popular (que incluye a los Bancos Popular y Pastor), al menos en lo que respecta a las cifras globales: crecimiento superior al 15 por 100 durante el primer subperiodo, debido sobre todo a la integración de algunas de las numerosas entidades con las que se fusionan (las más relevantes, en el caso de Sabadell, son Solbank, Herrero, Atlántico, CAM, Guipuzcoano o Gallego, mientras que en el Popular cabe mencionar a Crédito Balear, Castilla, Vasconia, Galicia o Andalucía) y fuerte caída a partir de ese año.

Destacar, finalmente, que Bankinter o Deutsche Bank (Gráfico 5) son las entidades que muestran un comportamiento más atípico dentro de los mayores bancos del

${ }^{6}$ Frente a la alternativa de representar en un solo gráfico cada grupo de entidades, hemos optado por agrupar aquellas que tienen un tamaño similar, con el fin de evitar que la mayor dimensión de algunas impida la visualización del patrón de comportamiento seguido por las de menor tamaño.

${ }^{7}$ Recuérdese que cuando se producen fusiones o adquisiciones entre entidades, para la construcción de las series que se presentan en esta sección se suman hacia atrás las oficinas de todas las entidades que forman parte de la operación corporativa.

${ }^{8}$ Como puede verse en dicho Gráfico 4, Banco Sabadell, en contra de la tendencia general, incrementa en 2013 su red en 561 oficinas (un aumento del 27 por 100). La explicación de esta aparente anomalía es que en ese año Sabadell incorpora la red de oficinas que Caixa Penedès (que, a pesar de esto, sigue formando parte de Banco Mare Nostrum) tiene en Cataluña y Aragón. 


\section{GRÁFICO 3}

\section{EVOLUCIÓN DE LA RED DE OFICINAS DE BANCO SANTANDER Y BBVA}

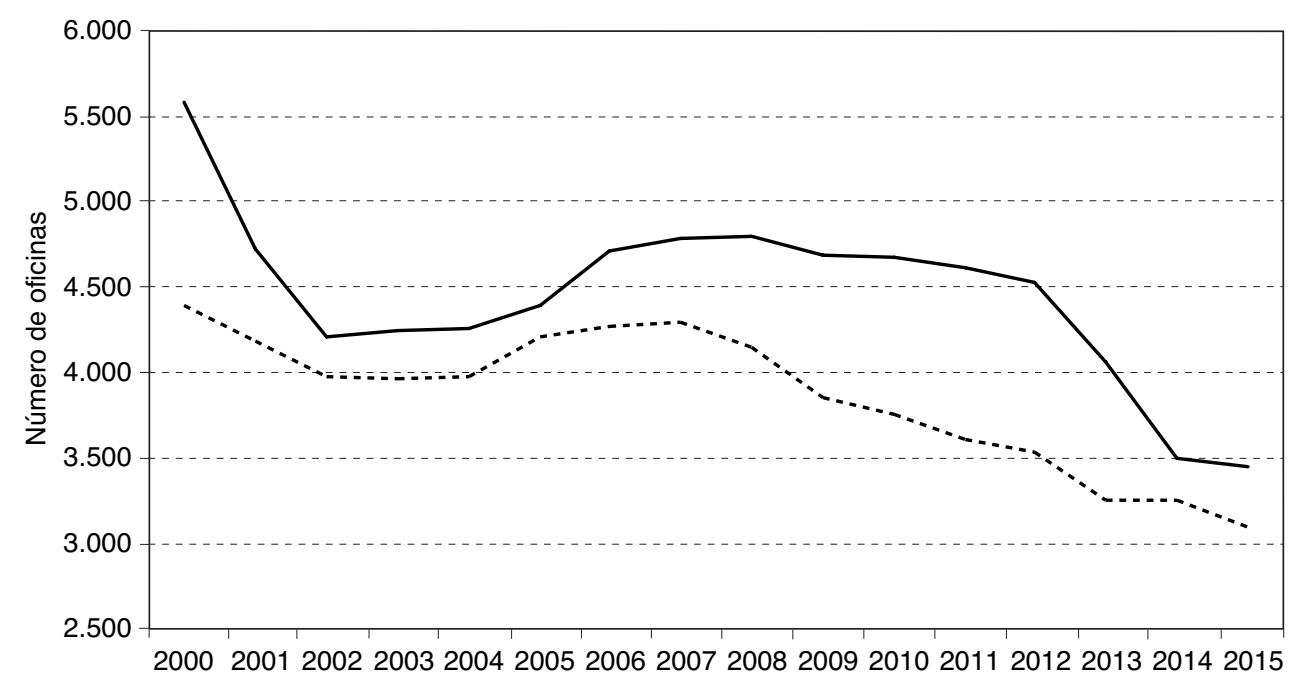

FUENTE: Elaboración propia.

— Banco Santander .... BBVA

\section{GRÁFICO 4}

\section{EVOLUCIÓN DE LA RED DE OFICINAS DE BANCO SABADELL Y GRUPO BANCO POPULAR}

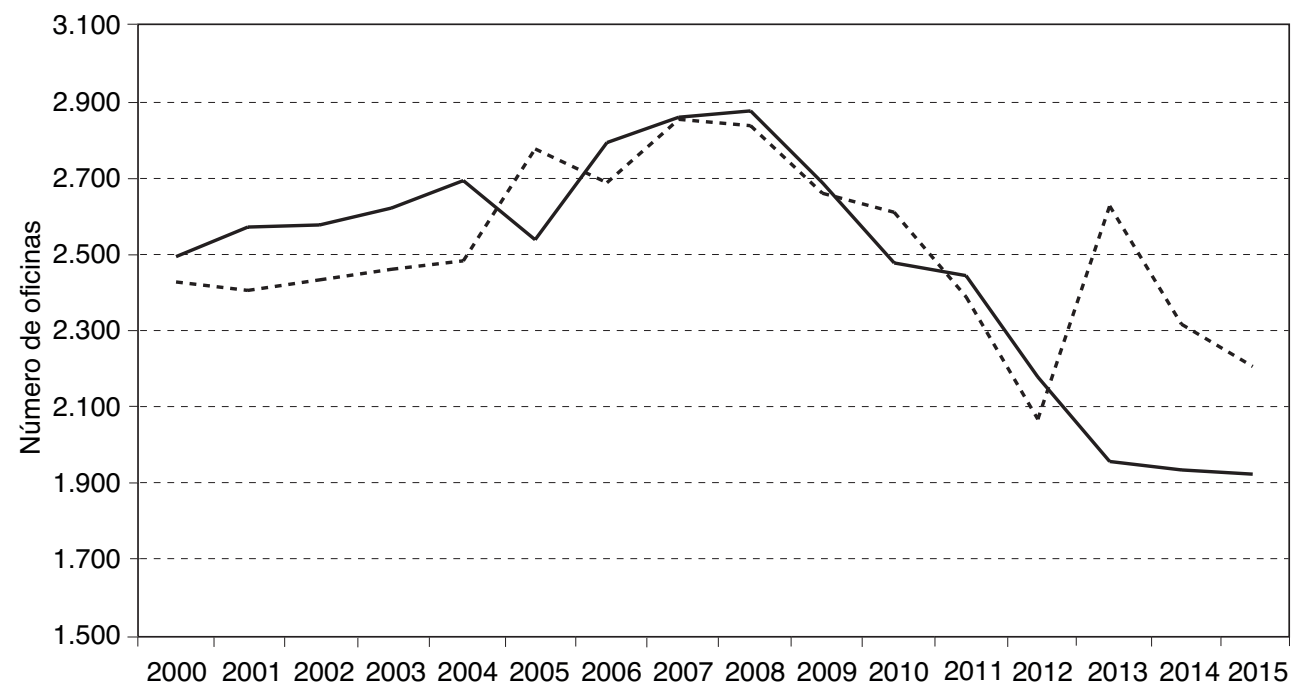

FUENTE: Elaboración propia.

$$
\text { --- Banco Sabadell _ Grupo Banco Popular }
$$




\section{GRÁFICO 5 \\ EVOLUCIÓN DE LA RED DE OFICINAS DE BANKINTER, DEUTSCHE BANK Y BANCA MARCH}

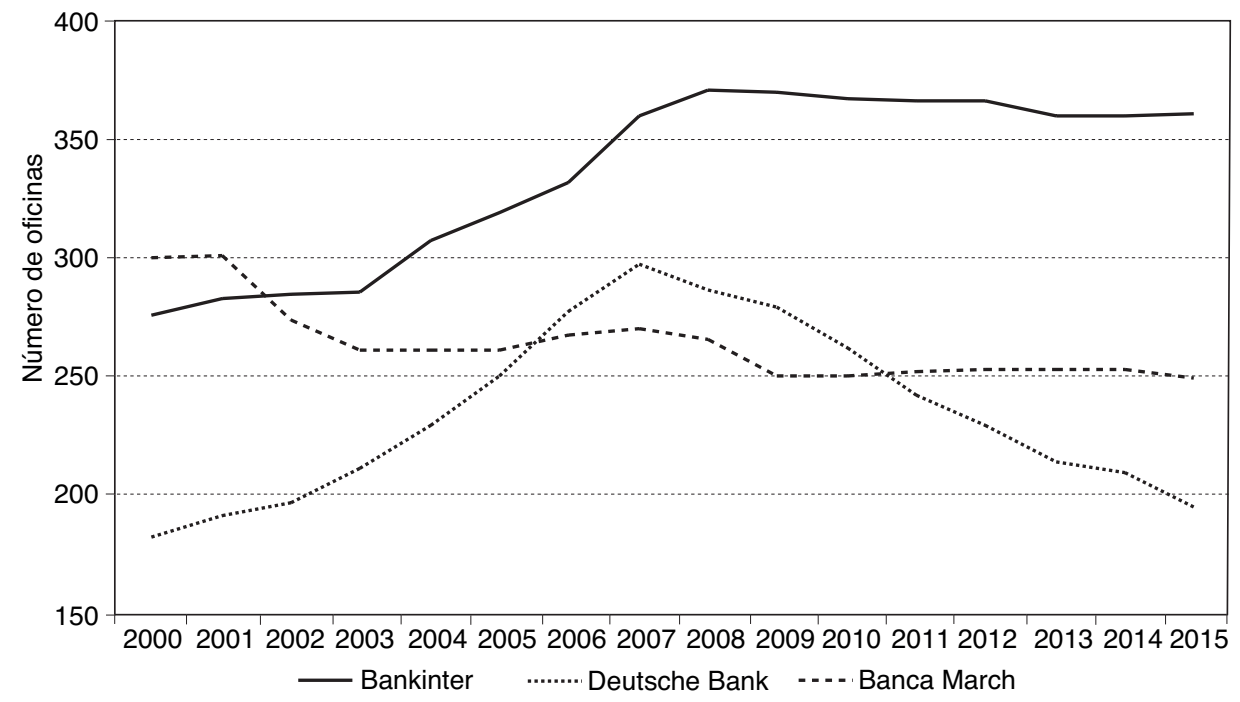

FUENTE: Elaboración propia.

país. Por lo que respecta al primero, aunque su red crece con fuerza hasta 2008 (un 34 por 100), apenas se reduce una vez iniciada la crisis: únicamente pierde 10 oficinas. En cuanto a Deutsche Bank, la reducción en el número de oficinas es moderada (17 por 100) y se distribuye a lo largo de los dos subperiodos analizados.

Por lo que respecta a las antiguas cajas de ahorro, los Gráficos 6,7 y 8 recogen la evolución de los principales grupos del país. En lo que hace referencia a las dos entidades líderes (Gráfico 6), su perfil en los años de crecimiento económico sostenido es parecido, con un aumento en el tamaño de la red en torno al 25-30 por 100. No resulta tan similar el patrón seguido por ambas a partir de 2008, ya que la caída en Caixabank es más regular y, aunque intensa, algo menos acusada (36,8 por 100), mientras que la de su rival no se inicia hasta la constitución en 2010 de BFA-Bankia, pero a partir de ese momento la red se reduce en más del 50 por 100, pasando de más de 4.000 oficinas en 2010 a solo 1.944 en 2015.

Según se muestra en el Gráfico 7, algo más prudente fue el crecimiento de Ibercaja durante los años previos a la crisis (un 17,7 por 100, únicamente originado como consecuencia de crecimiento orgánico) y también menos acusada la reestructuración posterior, ligeramente por encima del 25 por 100 y que se concentra en los años posteriores a la integración del grupo Caja3. Bastante similar es el comportamiento de Unicaja, aunque si se analizan los datos integrados con Caja España-Duero (téngase en cuenta que Unicaja la adquiere en 2014, aunque se mantienen ambas marcas, con la segunda operando fundamentalmente en Castilla y León) la caída en el segundo periodo es superior, ya que esta última cierra la mitad de sus oficinas. Tampoco 


\section{GRÁFICO 6}

\section{EVOLUCIÓN DE LA RED DE OFICINAS DE CAIXABANK Y BANKIA}

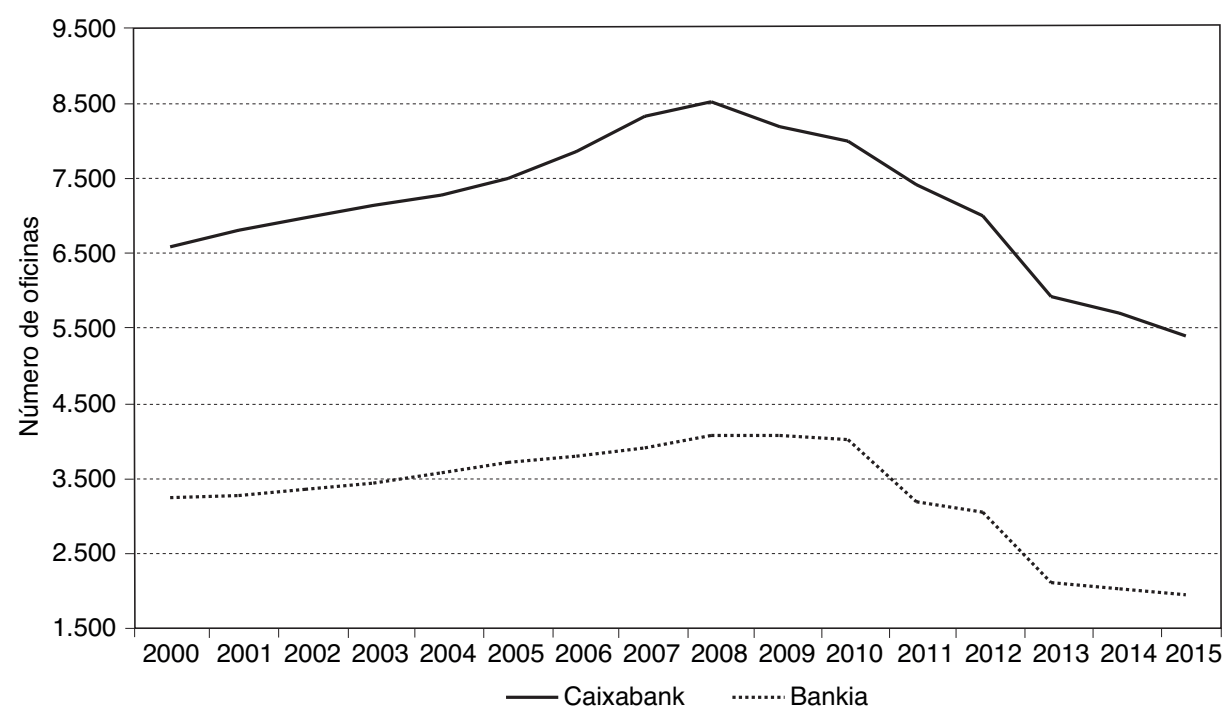

FUENTE: Elaboración propia.

\section{GRÁFICO 7}

EVOLUCIÓN DE LA RED DE OFICINAS DE IBERCAJA, UNICAJA (MÁS CAJA ESPAÑA-DUERO), BANCO MARE NOSTRUM, ABANCA Y GRUPO KUTXABANK

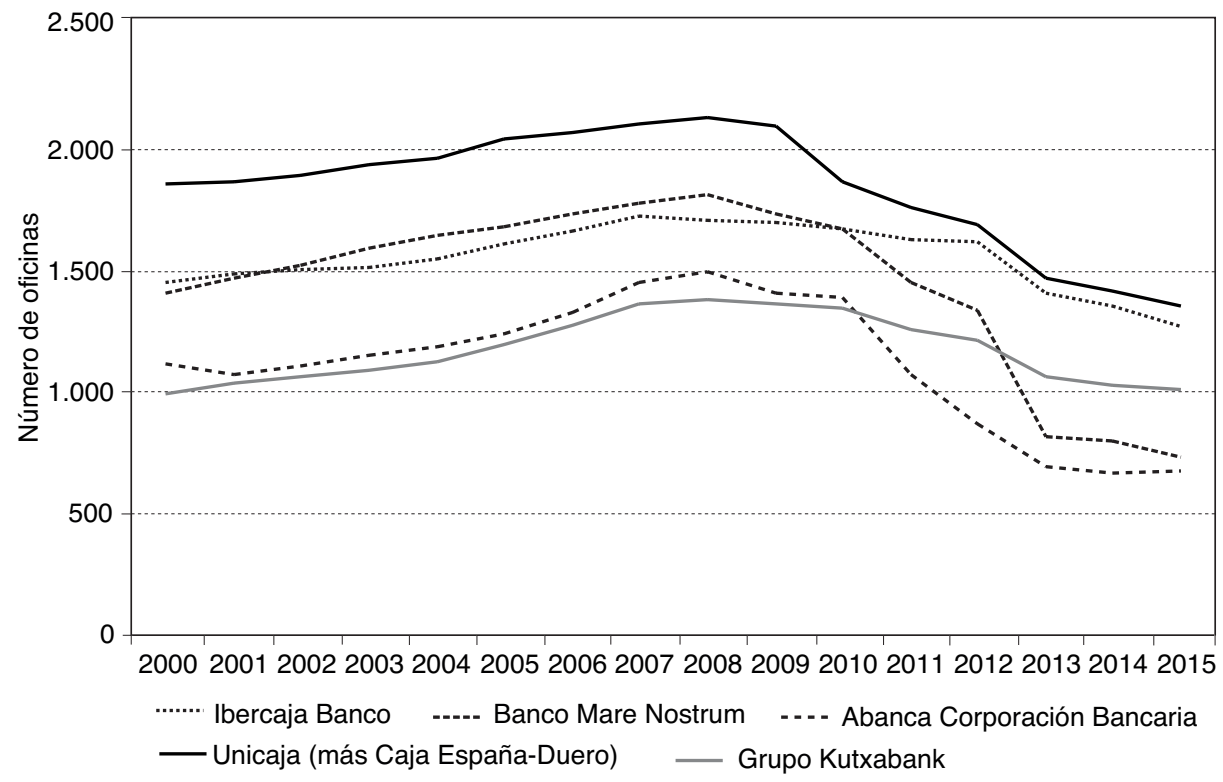

FUENTE: Elaboración propia. 


\section{GRÁFICO 8}

\section{EVOLUCIÓN DE LA RED DE OFICINAS DE LIBERBANK Y BANCO} DE CASTILLA-LA MANCHA

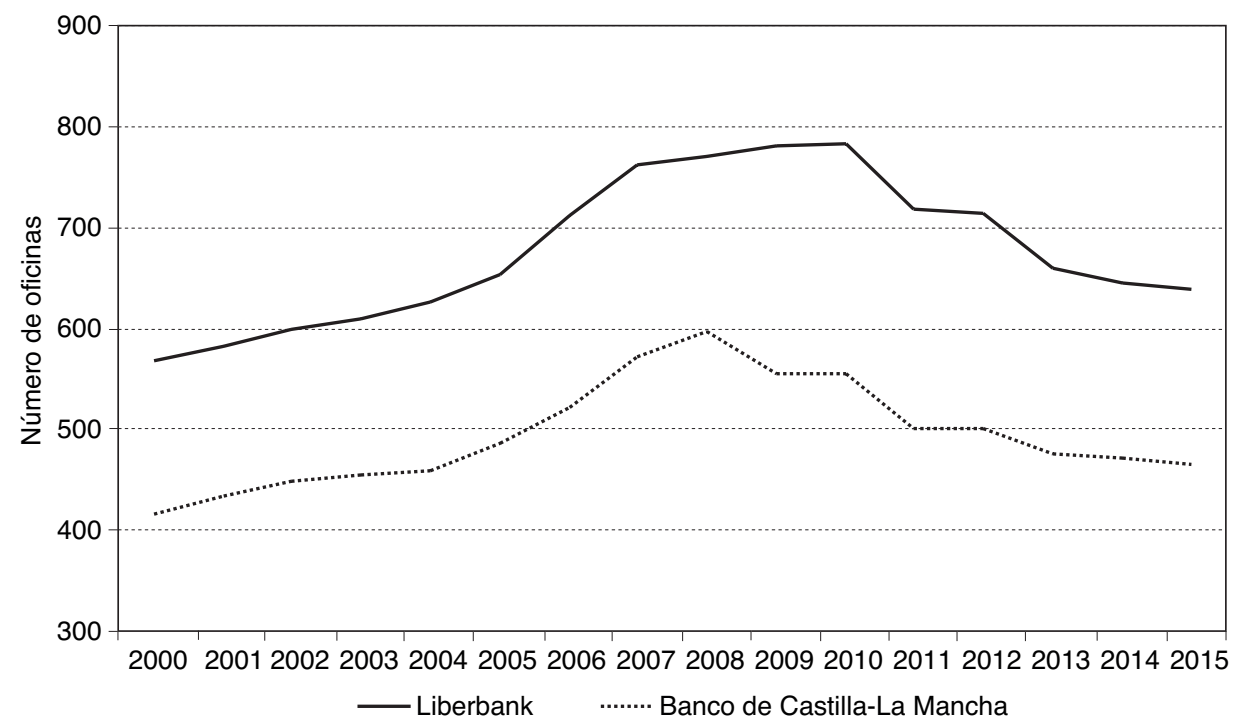

FUENTE: Elaboración propia.

difieren, en lo sustancial, las cifras de Kutxabank (la entidad resultante de las tres cajas vascas y que se adjudicó Cajasur en 2010, manteniendo la marca en Andalucía y Extremadura) una de las entidades que mejor ha superado el periodo de crisis, a pesar de que tras la fusión en 2011 elimina una de cada cuatro oficinas.

Resulta especialmente acusada la caída, a partir de 2008, en Abanca y Mare Nostrum, entidades originadas a partir de sendos procesos de fusión y que han atravesado por importantes dificultades en estos años. Así, Abanca (que integró en 2010 Caixanova y Caixa Galicia, y posteriormente el Banco Etcheverría) cierra un 55 por 100 de su red, mientras que la de Mare Nostrum (resultante de la fusión de las cajas de Murcia, General de Granada, Sa Nostra y Penedès) se reduce en un 60 por $100^{9}$.

Muy diferente ha sido, sin embargo, el comportamiento de las cajas rurales y cooperativas de crédito con la excepción, en todo caso, del grupo Cajamar (Gráfico 9), que también presenta un fuerte crecimiento inicial, que en esta entidad se extiende hasta 2010 como consecuencia de la integración de un buen número de cajas rurales y una caída, también importante, a partir de ese año. En el resto de las grandes cajas rurales y cooperativas de crédito, el fuerte crecimiento experimentado durante los

\footnotetext{
${ }^{9}$ Los Gráficos 6, 7 y 8 no hacen referencia a Catalunya Caixa debido a que la entidad es adquirida en la primavera de 2015 por BBVA. Sin embargo, la integración no se ha completado a finales de 2015, por lo que no se recoge en este análisis como parte integrante del grupo BBVA. No obstante, debemos mencionar que se trata de una de las entidades más golpeadas por la crisis, lo que le ha llevado a perder casi el 60 por 100 de sus oficinas.
} 


\section{GRÁFICO 9}

EVOLUCIÓN DE OFICINAS BANCO DE CRÉDITO COOPERATIVO (GRUPO CAJAMAR)

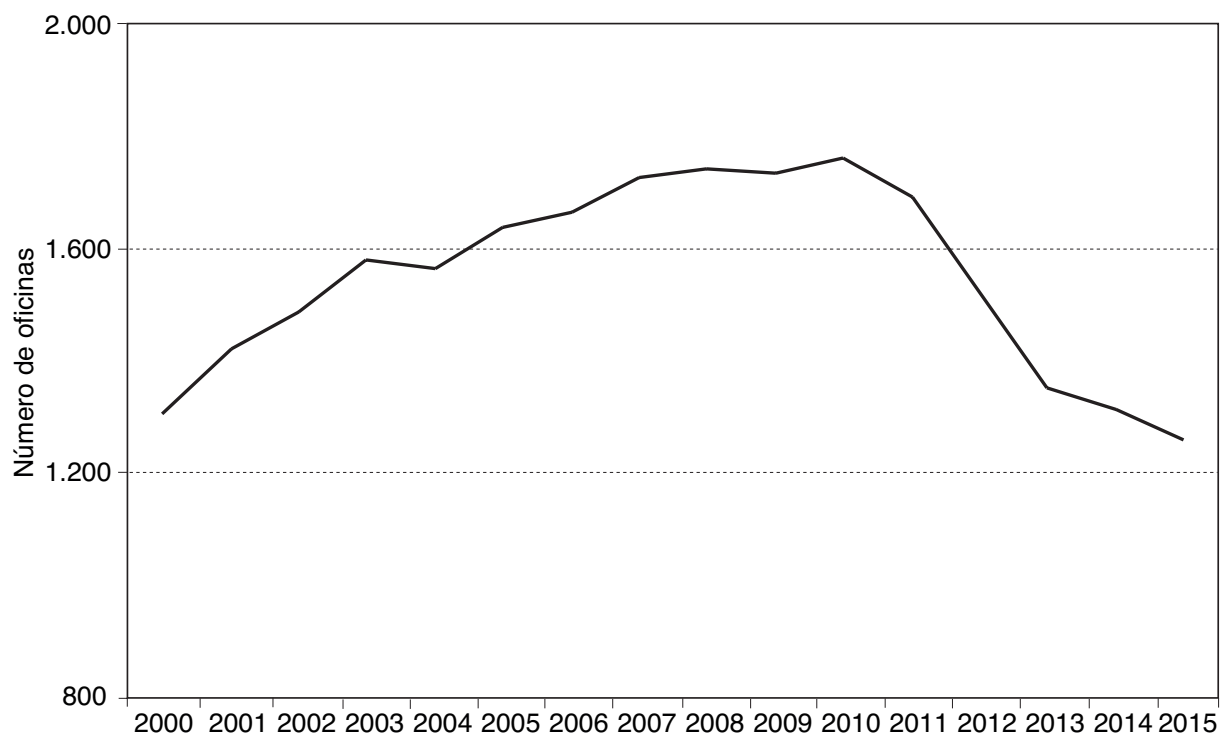

FUENTE: Elaboración propia.

\section{GRÁFICO 10}

EVOLUCIÓN DE LA RED DE OFICINAS DE CAJA LABORAL, BANTIERRA,

CAJA RURAL DE CASTILLA-LA MANCHA, CAJA RURAL DEL SUR, GLOBALCAJA Y CAJA RURAL DE NAVARRA

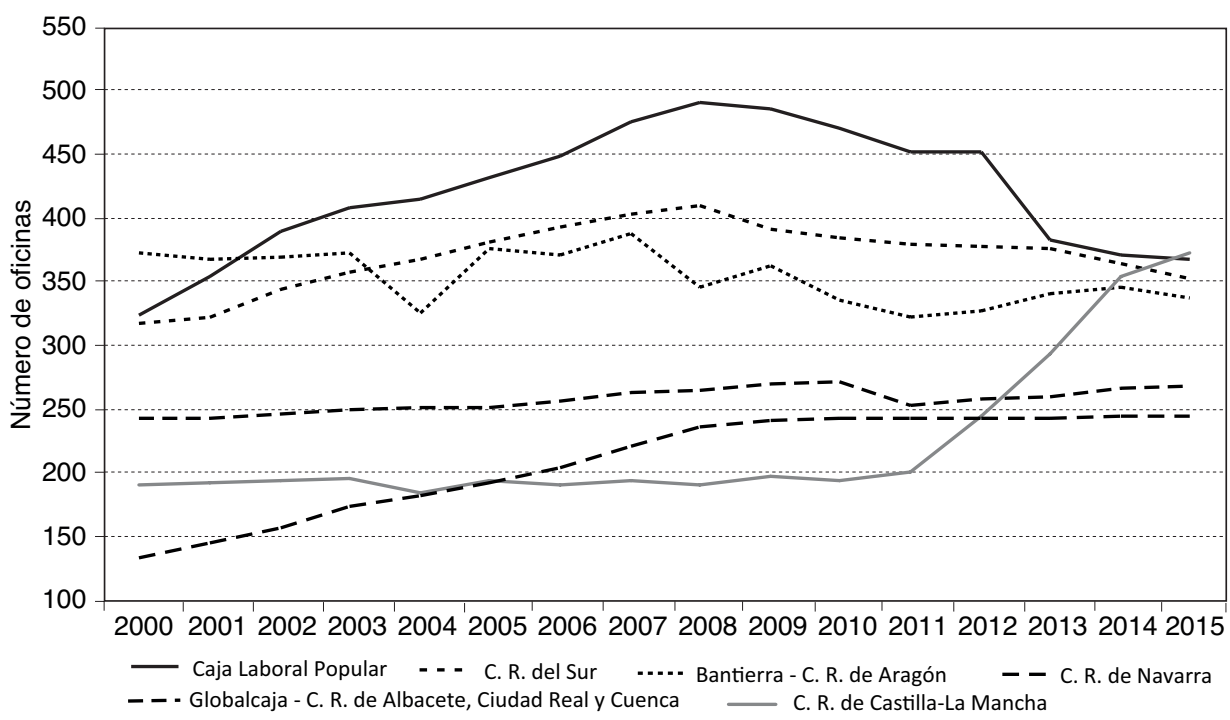

FUENTE: Elaboración propia. 
años iniciales (especialmente intenso en Caja Laboral o la Caja Rural de Navarra) se corrige de forma mucho más suave, hasta el punto de que algunas entidades (Globalcaja, CR Castilla-La Mancha) aumentan el tamaño de su red durante estos años de crisis, por lo que todas ellas cierran 2015 con más oficinas de las que tenían en 2000. La única excepción la constituye Bantierra, que reduce sus oficinas en la primera parte del horizonte temporal considerado.

\section{Factores determinantes de la evolución de la red de oficinas durante la crisis económica}

En el análisis descriptivo realizado en las dos secciones precedentes destacan dos aspectos. En primer lugar, durante la crisis económico-financiera se ha invertido el patrón de crecimiento que había tenido lugar en los años previos. Después de dos décadas de un aumento sostenido, el número de oficinas sufre, desde 2008, una reducción sin precedentes. En segundo lugar, esta evolución no se da de manera homogénea entre todas las entidades financieras. Por ejemplo, las cajas rurales han reducido su red de forma mucho más moderada que los bancos comerciales o las cajas de ahorros. A su vez, determinadas entidades se han visto menos afectadas por este proceso. En consecuencia, resulta interesante profundizar en las causas específicas que han llevado a esta situación, especialmente en aquellas que pueden ayudarnos a entender las diferencias que se observan entre entidades.

En concreto, se discute aquí el efecto de tres tipos de factores. En primer lugar, se hará referencia a dos factores externos a las empresas: la reducción en el volumen de actividad que se produce durante la crisis y la evolución tecnológica. En segundo lugar, se proponen explicaciones basadas en el comportamiento estratégico de las empresas. Entre estos factores se incluyen la estrategia de expansión de la red de oficinas seguida durante el periodo de expansión previo a la crisis, las fusiones y adquisiciones que han tenido lugar durante el periodo observado y las diferencias sistemáticas en la misión y objetivos estratégicos que se dan entre los diferentes tipos de intermediarios que operan en el sistema bancario español. En tercer lugar, discutiremos el efecto del desempeño de las empresas. Particularmente, haremos referencia al desempeño operativo (eficiencia).

Estos factores pueden ayudar a entender, siquiera parcialmente, la reducción en la red que tiene lugar a partir de 2008. Comenzaremos con una breve descripción de cada uno de estos elementos y un primer análisis exploratorio. Después, llevaremos a cabo un análisis de descomposición de la varianza de la propensión al cierre de oficinas con el fin de evaluar la importancia relativa de estos elementos en la evolución de la red de oficinas ${ }^{10}$.

\footnotetext{
${ }^{10}$ Cabe destacar que todos estos análisis se refieren al periodo 2008-2015, es decir, desde el comienzo de la crisis y hasta la información más actualizada que ofrecen los anuarios de AEB, CECA y UNACC.
} 
En estos análisis se mide la propensión al cierre como el porcentaje de oficinas cerradas durante un año (número de oficinas al comienzo del periodo menos número de oficinas a final de año dividido por el número inicial de oficinas) ${ }^{11}$. La muestra incluye todas las entidades bancarias que operaron en España durante el periodo y que en algún momento alcanzaron al menos las 10 oficinas. Este requisito mínimo de oficinas se aplica para evitar sobrevalorar el impacto de los cierres de oficinas en entidades muy pequeñas, y para asegurar que en las entidades analizadas la banca minorista tiene un papel relevante.

\subsection{Reducción de actividad bancaria}

Uno de los efectos más importantes de la crisis económica fue una notable reducción en el volumen de actividad económica. Esta reducción se trasladó al sector financiero, en forma de menor demanda de créditos y menor volumen de depósitos en los bancos. En tanto que las oficinas son la unidad básica de provisión de los servicios asociados a operaciones tanto de crédito como depósito, la red de oficinas existente pasó a estar sobredimensionada. En consecuencia cabe esperar que se produzca una variación en la red de oficinas análoga a la variación en la demanda de estos servicios.

El Gráfico 11 proporciona una primera aproximación a este fenómeno. En él se muestra la evolución de la red de oficinas (eje de la izquierda) y del volumen total de créditos y depósitos (eje de la derecha, en millones de euros de 1991). Desde 2008, la reducción en la red de oficinas ha sido más acusada que la reducción en el volumen de negocio. Más aún, entre 2012 y 2015 se observa cierta moderación en la caída del volumen de actividad; sin embargo, la red de oficinas continúa disminuyendo con fuerza. En definitiva, la evolución del volumen de actividad que, en consonancia con el interés en las actividades de banca minorista que nos mueve en este análisis, aproximamos mediante la suma del total de créditos y depósitos de cada entidad, proporciona, en todo caso, una explicación parcial. No obstante, se puede realizar un análisis más robusto teniendo en cuenta las especificidades de cada entidad. Particularmente, comparamos la propensión al cierre de oficinas por parte de las entidades financieras en función de la variación en el volumen de actividad que experimentan.

Para analizar el efecto de una reducción en el volumen de actividad, separamos las entidades en función de si en un determinado ejercicio experimentan un aumento notable en su volumen de actividad (i.e., variación mayor que la media del sector más una desviación típica), muestran una cierta estabilidad (variación en un intervalo

\footnotetext{
${ }^{11}$ Nos centramos en analizar los factores que explican la reducción de la red de oficinas. Por tanto, esta medida no recoge los casos en los que una entidad aumenta de forma neta su tamaño. Una entidad en la que aumente el número de oficinas activas tendrá un valor de 0 en la medida de propensión al cierre. En el caso de entidades fusionadas, comparamos las oficinas de las entidades implicadas con las oficinas de la entidad resultante.
} 


\section{GRÁFICO 11}

EVOLUCIÓN DE LA RED. EVOLUCIÓN DE LA RED DE OFICINAS BANCARIAS Y DEL VOLUMEN DE ACTIVIDAD

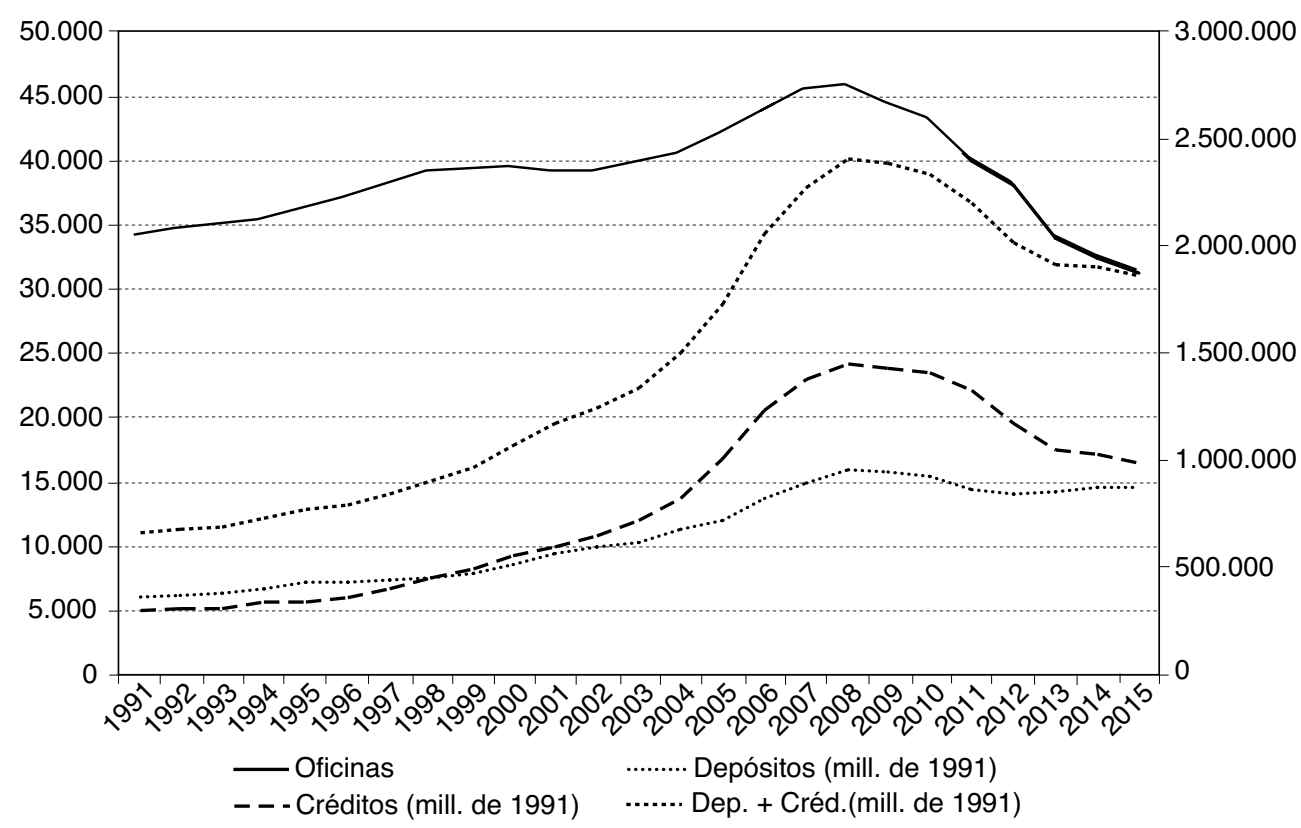

FUENTE: Elaboración propia.

de la media más, o menos, una desviación típica), o experimentan una reducción notable de actividad en relación con el año anterior (media del sector menos una desviación típica), y analizamos la tasa de cierre de oficinas en el siguiente ejercicio.

Entre las entidades que experimentan un descenso notable en el volumen de actividad, la tasa promedio de cierre de oficinas ha sido de un 12 por 100 anual, en claro contraste con las entidades para las cuales el volumen de actividad permanece estable o aumenta de forma significativa (en ambos casos, propensión al cierre por debajo del 4 por 100). Un test ANOVA de diferencia de medias entre estas tres categorías (aplicando la corrección de Bonferroni) confirma que estas diferencias resultan estadísticamente significativas $(\mathrm{F}=12,41 ; \mathrm{p}<0,001)$. Por tanto, las variaciones en el volumen de demanda constituyen una explicación plausible del elevado cierre de oficinas observado entre 2008 y 2015.

\subsection{Evolución tecnológica}

Un segundo factor que podría explicar la variación en la red de oficinas es el cambio tecnológico. Desde la década de los 80 y coincidiendo con la liberalización del sector bancario, se han producido numerosas innovaciones destinadas a la 
automatización y la mejora de eficiencia de las actividades bancarias, con especial apoyo en las tecnologías de la información (Batiz-Lazo y Woldesenberg, 2006; Boot y Marinç, 2008). Entre estos cambios, cabe destacar la computarización de las actividades administrativas, la coordinación de sistemas de balances, la consolidación de los terminales en el punto de venta y los cajeros automáticos, o el desarrollo de la banca online. Estas iniciativas presentan normalmente economías de escala, por lo que han podido aumentar la capacidad de prestar servicio de cada oficina. Como resultado, el cierre de oficinas de la última década podría haber sido, al menos parcialmente, una consecuencia de ese proceso de sustitución entre tecnología y oficinas.

El Gráfico 12 muestra el volumen de créditos y depósitos que se gestionan desde cada oficina en promedio (en millones de euros de 1991). Un hecho admitido en la literatura sobre cambio tecnológico es que las mejoras en el desempeño como consecuencia de la introducción de nuevas tecnologías suelen mostrar un patrón en forma de «S», de modo que la contribución de la tecnología es moderada pero creciente tras su introducción, aumenta a continuación de forma más rápida, para finalizar con mejoras marginales como resultado de la saturación de las oportunidades tecnológicas (e.g., Nieto, López y Cruz, 1998). En consecuencia, en caso de que el cambio tecnológico haya tenido un impacto tangible en la actividad de las entidades bancarias españolas, en las últimas décadas cabría esperar un aumento en forma de curva sigmoidal en la capacidad de servicio de cada oficina.

En el Gráfico 12 se observa que el volumen de actividad asumido por cada oficina muestra la forma de «S» discutida en el párrafo anterior (que analíticamente se traduce en una relación). Este patrón es consistente con los años de introducción paulatina de nuevas aplicaciones de las tecnologías de la información en el sector. En cuanto al efecto de la crisis, cabe destacar que la actividad de cada oficina ha aumentado sostenidamente desde los años 90, esto es, desde mucho antes de la crisis. La línea vertical corresponde al año 2008, cuando se alcanzó el máximo de oficinas de la red. Es importante señalar que las series no parecen experimentar una discontinuidad localizada en ese año: el volumen de actividad llevado a cabo por cada oficina va aumentando con el mismo patrón de años anteriores. Esto podría suponer que la reducción de la red de oficinas responde no solo a las variaciones en el volumen de actividad a llevar a cabo, sino a la evolución tecnológica y su impacto en el volumen de actividad que se puede llevar a cabo desde cada oficina.

Para comprobar de manera más formal el posible efecto del cambio tecnológico en la propensión al cierre de oficinas, llevamos a cabo un test de comparación de medias (ANOVA, corrección Bonferroni). Téngase en cuenta que el sector bancario se caracteriza por su dependencia de proveedores externos para obtener innovaciones tecnológicas. Como resultado, existe mayor homogeneidad en el acceso a nuevas tecnologías del que pueda existir en otros sectores. Esto permite tratar el cambio tecnológico como un efecto exógeno común. Así, comparando la propensión al cierre de oficinas de cada año, podemos aproximar el efecto previsible del desarrollo tecnológico. El test rechaza la hipótesis nula de que existen diferencias sistemáticas en el cierre de oficinas anuales $(F=1,26, p=0,273)$. En consecuencia, el patrón 


\section{GRÁFICO 12}

VOLUMEN DE CRÉDITOS Y DEPÓSITOS POR OFICINA

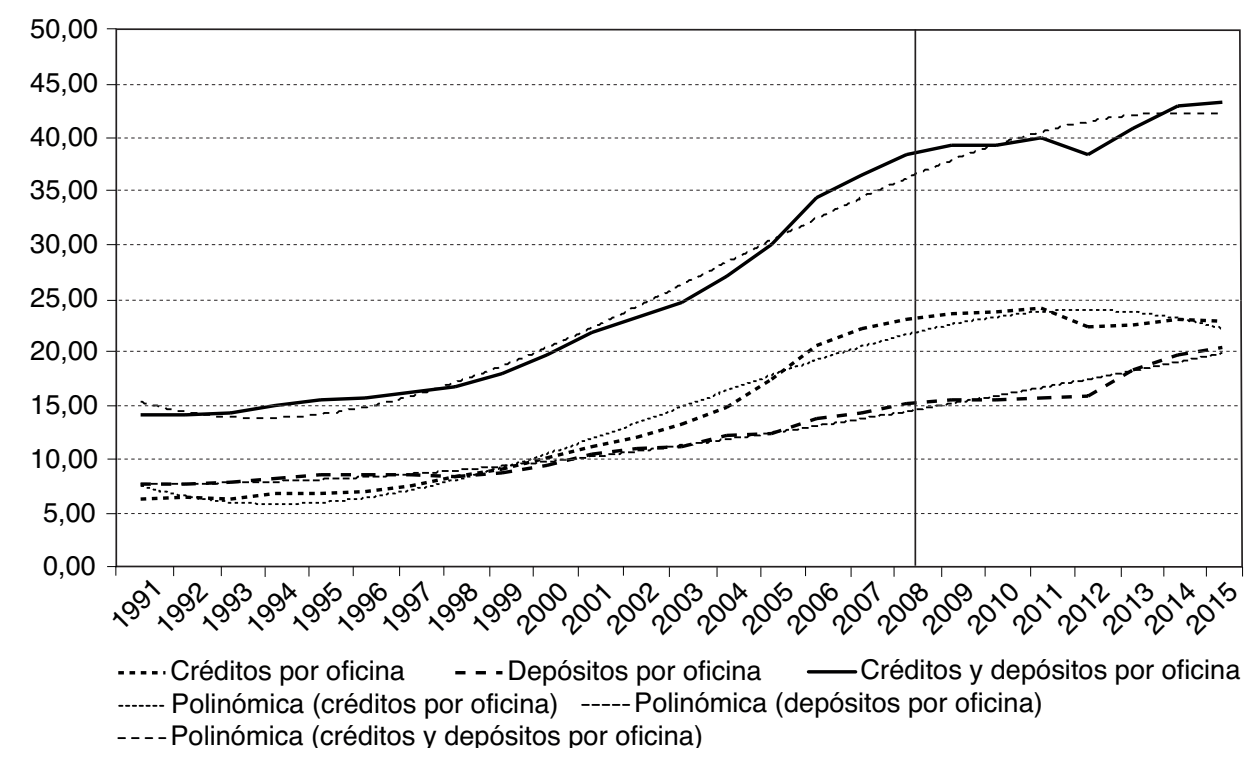

FUENTE: Elaboración propia.

observado no parece consistente con un patrón de cierre de oficinas basado fundamentalmente en el cambio tecnológico ${ }^{12}$.

\subsection{Estrategias de crecimiento durante el periodo de expansión}

Entrando ya en las particularidades de la estrategia seguida por cada empresa, otra explicación para la reducción de la red de oficinas sería la estrategia de crecimiento adoptada por cada entidad durante los años de desarrollo económico sostenido que tuvieron lugar entre 2000 y 2008. Durante este periodo muchas entidades se embarcan en estrategias de crecimiento agresivo que podrían haber sobredimensionado el número de puntos de venta. En el nuevo escenario económico las entidades, conscientes de este exceso, podrían estar corrigiendo las decisiones adoptadas en años anteriores. En la medida en que este argumento pudiera estar detrás de la disminución en el tamaño de la red, aquellos bancos cuyo número de oficinas hubiese crecido por encima del volumen de actividad del mercado estarían más expuestos a esta corrección.

\footnotetext{
${ }_{12}$ Nos centramos en analizar los factores que explican la reducción de la red de oficinas. Por tanto, esta medida no recoge los casos en los que una entidad aumenta de forma neta su tamaño. Una entidad en la que aumente el número de oficinas activas tendrá un valor de 0 en la medida de propensión al cierre. En el caso de entidades fusionadas, comparamos las oficinas de las entidades implicadas con las oficinas de la entidad resultante.
} 
Para analizar este argumento, comparamos la propensión al cierre de oficinas de las entidades en función de su estrategia de crecimiento durante el periodo de expansión. Las empresas cuya red de oficinas creció un 50 por 100 más rápido que el volumen total de créditos nacional las etiquetamos como empresas que siguieron una estrategia agresiva. Las entidades cuya red creció hasta un 50 por 100 más lentamente que el volumen de actividad del sector las etiquetamos como empresa que siguieron una estrategia defensiva y al resto las catalogamos como empresas con una estrategia neutra. La propensión al cierre de oficinas en cada categoría es de un 4,9 por 100 , un 3,3 por 100 y un 10 por 100 para las estrategias defensiva, neutra y agresiva respectivamente. Estas diferencias son estadísticamente significativas $(\mathrm{F}=8,71, \mathrm{p}<0,001)$. Por tanto, las empresas que durante el periodo de expansión económica desarrollaron su red de oficinas a mayor ritmo son las que en los años siguientes experimentaron una mayor propensión al cierre de oficinas.

\subsection{Fusiones y adquisiciones}

Entre 2008 y 2015 se han producido numerosas operaciones de fusión y adquisición entre entidades bancarias. Algunas operaciones pueden haber sido propiciadas por el regulador, en un intento de consolidar y estabilizar el sistema. Otras pueden ser el resultado de la identificación de una oportunidad por parte de las entidades. En cualquier caso, las fusiones suelen generar problemas organizativos y potenciales solapamientos en actividad y alcance geográfico que derivan en el cierre de oficinas.

Para profundizar en este argumento, analizamos la propensión al cierre de oficinas de cada entidad el año siguiente de verse implicada en fusiones y adquisiciones en función de la importancia de la operación. En concreto, identificamos tres grupos. En el primero se incluyen aquellos bancos o cajas que no se han visto implicados en ninguna fusión en el año anterior. El segundo engloba las entidades en las que estas operaciones corporativas han generado un aumento en su red de oficinas de menos de un 10 por 100. Por último, se consideran los intermediarios en los que su red se ve incrementada más de un 10 por 100. Para calcular este aumento, nos fijamos en el periodo previo a la operación. Si la entidad resultante de la operación ya existía (según el código del banco de España), tomamos a esta como referencia. Si se crea una entidad nueva, suponemos que la entidad de mayor tamaño entre las implicadas es la que ve variar su red de oficinas, mientras que las otras entidades desaparecen. Cuando una entidad se enfrenta a una fusión o adquisición de mayor tamaño relativo, mayor es el potencial desajuste en sus operaciones y, en consecuencia, la posibilidad de cierre de oficinas en los años siguientes.

Los resultados muestran que la propensión al cierre entre las entidades que no participan en estas operaciones es de un 3,7 por 100, cifra inferior a la de las entidades involucradas en operaciones en las que el tamaño de la absorbida es inferior al 10 por 100 (6,5 por 100 de cierre de oficinas) y lejos de las entidades involucradas en operaciones superiores al 10 por 100 (9,1 por 100 de cierres). Estas diferencias 
son estadísticamente significativas $(\mathrm{F}=3,35 ; \mathrm{p}=0,036)$, por lo que los resultados sugieren que las fusiones y adquisiciones tuvieron un peso notable en la propensión al cierre de oficinas tras la crisis.

\subsection{Diferencias en misión y modelo de negocio}

Un último factor relacionado con la estrategia de cada entidad financiera tiene que ver con la naturaleza de sus objetivos. En el sector bancario, los tres tipos tradicionales de agente han tenido históricamente diferentes misiones y modelos de negocio. Mientras que la banca comercial estaba dirigida a promover el desarrollo industrial y centraba tradicionalmente su actividad en proporcionar recursos a las empresas de cierta dimensión, los fines de las cajas de ahorro y las cooperativas tenían un componente más social, priorizando aspectos como el fomento del ahorro, la financiación del consumo, el apoyo a las pymes o garantizar la inclusión financiera. Aunque la liberalización que tuvo lugar durante los años ochenta del siglo pasado permitía a todas las entidades realizar cualquier tipo de actividad, eliminando las restricciones legales existentes, las entidades siguieron manteniendo, al menos en lo esencial, sus modelos de negocio durante un largo periodo, por lo que es de esperar que las diferencias estratégicas entre estas entidades se sigan manteniendo debido a los valores organizativos tradicionales de cada forma organizativa.

Estos valores implicarían que cajas y cooperativas podrían ser más reacias a cerrar oficinas, en la medida en la que prioricen valores como la provisión de servicios a determinados colectivos o la inclusión financiera frente a los objetivos estrictamente de resultados. En el Gráfico 2 se observaba cómo había diferencias sistemáticas entre cada una de las formas organizativas, tanto desde la liberalización del sector como en el punto de inflexión que se produce con la crisis. Sin embargo, se trata de análisis agregados. Con el fin de profundizar en estas ideas llevamos a cabo un análisis individual analizando cómo la propensión al cierre de oficinas varía en función de la forma organizativa de cada entidad. Particularmente, la propensión al cierre en bancos, cajas y cooperativas es del 6,1 por $100,5,2$ por 100 y 1,2 por 100 respectivamente. Estas diferencias son estadísticamente significativas $(F=13,15, p<0,001)$, por lo que la naturaleza de cada tipo de entidad parece explicar también parte de las diferencias en el cierre de oficinas observado desde 2008.

\subsection{Diferencias en desempeño}

Un último tipo de factores que podrían explicar la heterogeneidad en la propensión al cierre de oficinas es el desempeño de las empresas. Ante problemas de rendimiento, reducir el tamaño cerrando aquellas oficinas menos eficientes puede ser una respuesta apropiada. En consecuencia, empresas con problemas de desempeño operativo podrían mostrar una mayor propensión al cierre. 
Analizamos aquí el desempeño operativo (eficiencia) a través del cociente entre gastos de administración y margen bruto y creamos tres grupos en función de si este ratio tiene un valor superior a la media del año más una desviación típica (menos eficientes), si tiene un valor inferior a la media del año menos una desviación típica (eficientes), o si su valor se encuentra a una distancia de la media del año inferior a una desviación típica (eficiencia media). Estos grupos muestran una propensión al cierre del 4,5 por $100,3,4$ por 100 y 5,5 por 100 respectivamente, valores que no resultan estadísticamente diferentes $(F=2,15 ; p=0,117)$, lo que sugiere que los intentos por mejorar los niveles de eficiencia no parecen estar detrás de la reducción observada en la red de oficinas.

\subsection{Análisis de componentes de la varianza}

Los análisis descriptivos presentados hasta el momento proporcionan una primera aproximación a los principales factores que explican la propensión al cierre de oficinas de cada entidad. Sin embargo, estos análisis presentan dos limitaciones importantes. En primer lugar, al adoptar una aproximación univariante no tienen en cuenta la correlación que existe entre los diferentes factores. En consecuencia, no es posible asegurar que el efecto observado corresponde estrictamente a cada variable o si, por el contrario, está contaminado por el efecto de otras variables que no se han tenido en consideración. En segundo lugar, a partir de los test de comparación de medias se puede estudiar si cada categoría muestra una propensión al cierre diferente, pero no se analiza la importancia relativa de cada uno de los factores estudiados.

Con el fin de resolver estos problemas, a continuación se lleva a cabo un Análisis de Descomposición de la Varianza que incluye en el mismo modelo todos los factores discutidos. Más concretamente, utilizamos un análisis de Máxima Verosimilitud que hace uso de las categorías discretas propuestas para cada factor como grupos alternativos en cada uno de estos factores. Así, podemos estimar qué porcentaje de la variabilidad observada en la muestra se ve explicada por cada variable, controlando por las interdependencias entre cada uno de estos factores. El Cuadro 2 muestra los resultados del análisis. En ella se observa que los factores propuestos explican en torno al 50 por 100 de la varianza en la propensión al cierre de oficinas de las entidades españolas durante la crisis. La varianza residual podría achacarse a otras variables omitidas y al efecto idiosincrásico de cada entidad.

Como cabía esperar, los factores que no resultaban significativos en el test ANOVA son los que explican una menor proporción de la varianza. La eficiencia explica solo un 1,41 por 100 de la varianza total (2,79 por 100 de la varianza explicada) y la evolución tecnológica, un 4,18 por 100 (8,26 por 100 de la varianza explicada). De entre los factores que se identificaron como significativos, el que tiene mayor poder explicativo es la variación en el volumen de demanda de servicios financieros. Esta variable por sí sola explica el 16,02 por 100 de la varianza en la propensión al cierre de oficinas, representando casi un tercio de la varianza explicada por el conjunto de 


\section{CUADRO 2}

ANÁLISIS DE COMPONENTES DE LA VARIANZA

\begin{tabular}{|l|c|c|c|}
\hline \multicolumn{1}{|c|}{ Factor } & Varianza & $\begin{array}{c}\text { Porcentaje sobre } \\
\text { total }\end{array}$ & $\begin{array}{c}\text { Porcentaje sobre } \\
\text { varianza explicada }\end{array}$ \\
\hline Demanda & 0,0350 & 16,02 & 31,62 \\
\hline Tecnología & 0,0091 & 4,18 & 8,26 \\
\hline Expansión & 0,0214 & 9,78 & 19,31 \\
\hline Fusiones & 0,0217 & 9,90 & 19,55 \\
\hline Misión & 0,0205 & 9,36 & 18,47 \\
\hline Eficiencia & 0,0031 & 1,41 & 2,79 \\
\hline Residual (no explicada) & 0,1079 & 49,35 & - \\
\hline
\end{tabular}

FUENTE: Elaboración propia.

factores. Los tres factores restantes (estrategia de crecimiento seguida durante los años de expansión, misión propia de cada tipo de entidad y fusiones y adquisiciones) explican cada uno algo más del 9 por 100 de la varianza, lo que supone aproximadamente un 19 por 100 de la varianza explicada por el modelo.

\section{Conclusiones}

El objetivo de este trabajo ha sido identificar, de forma descriptiva, los principales factores que determinan el proceso de cierre de oficinas bancarias que ha tenido lugar en España entre 2008 y 2015. Es indudable que una parte de estos cierres puede estar justificada por la caída en la actividad que se deriva de la reciente crisis económica, pero no parece que esta sea la única explicación a esta reestructuración. En esta investigación hemos propuesto seis posibles causas explicativas: contracción de la demanda, desarrollo tecnológico, estrategias de crecimiento seguidas durante el periodo de expansión, fusiones y adquisiciones, diferencias en la misión o el modelo de negocio o diferencias en el grado de desempeño (eficiencia) de las entidades.

A través de un análisis de descomposición de la varianza hemos observado que dos de estos motivos (progreso tecnológico y diferencias en los niveles de eficiencia entre entidades) parecen haber tenido un efecto marginal en la explicación de la varianza observada. Del resto de factores esgrimidos, el más importante es la caída de la demanda de servicios bancarios, que explica 16 por 100 de la varianza total (casi una tercera parte del total de la varianza explicada). El resto de argumentos explican individualmente en torno al 9-10 por 100. Destacar, por último, que el modelo explica en conjunto aproximadamente la mitad (49,35 por 100) de la varianza total.

Evidentemente, la aproximación realizada en este trabajo presenta algunas limitaciones, que deberán ser objeto de un análisis más detallado en futuras investigaciones. Como hemos apuntado, el análisis adopta un enfoque eminentemente 
descriptivo, en el que únicamente se analiza la correlación que existe entre el cierre de oficinas y algunas de sus posibles causas. No se han ofrecido, sin embargo, argumentos teóricos que justifiquen la elección de estas variables, más allá de que la literatura o las propias valoraciones realizadas por parte de los directivos u otros expertos en la industria hayan hecho con frecuencia mención a las mismas. En consecuencia, el desarrollo de un modelo teórico que profundice en las causas de dichos cierres, completado con un análisis multivariante que integre dichas razones en un mismo modelo, proporcionaría una mayor solidez a los resultados aquí obtenidos.

Es importante también tener en cuenta que este trabajo no analiza la validez teórica o empírica de estos factores, sino si en el contexto específico de España, y durante la crisis económica que comienza en 2008, han estado detrás de la notable reducción en la red de oficinas que se observa. Cabe destacar que estos factores ofrecen explican aproximadamente la mitad de la varianza en relación con el número de cierres de oficinas bancarias. En consecuencia, seguramente existen otros motivos adicionales a los considerados en esta investigación, por lo que también sería de interés identificar cuáles pueden ser estas razones.

\section{Referencias bibliográficas}

[1] BATIZ-LAZO, B. y WOLDESENBET, K. (2006). «The dynamics of product and process innovations in UK banking». International Journal of Financial Services Management, 1, 400-421.

[2] BOOT, A. W. A. y MARINÇ, M. (2008). «The evolving landscape of banking». Industrial and Corporate Change, 17, 1173-1203.

[3] DELGADO, J.; SAURINA, J. y TOWNSEND, R. (2008). «Estrategias de expansión de las entidades de depósito españolas. Una primera aproximación descriptiva». Estabilidad Financiera, 15, 99-117.

[4] FUENTELSAZ, L.; GÓMEZ, J. y PALOMAS, S. (2007). «La reestructuración de la red de oficinas en el sector bancario español: 1995-2005». Papeles de Economía Española, 114, 173-187.

[5] FUENTELSAZ, L.; GÓMEZ, J. y POLO, Y. (2004). «La expansión de la red de oficinas de las cajas de ahorro: una perspectiva estratégica». Perspectivas del Sistema Financiero, 80, 57-71.

[6] MALO DE MOLINA, J. L. y MARTÍN-ACEÑA, P. (eds.) (2011). Un siglo de historia del sistema financiero español, Alianza Editorial, Madrid.

[7] MAUDÓS, J. (2017). «El acceso a los servicios bancarios en España el impacto de la reducción del número de oficinas». Mediterráneo Económico, 29, 265-285.

[8] MAUDÓS, J. (2018). «Bank restructuring and access to financial services: the Spanish case». Growth and Change (en prensa).

[9] NIETO, M.; LÓPEZ, F. y CRUZ, F. (1998). «Performance analysis of technology using the $\mathrm{S}$ curve model: the case of digital signal processing (DSP) technologies». Technovation, 18, 439-457. 